\title{
Correction to: Gallai-Ramsey Numbers for a Class of Graphs with Five Vertices
}

\section{Xihe $\mathrm{Li}^{1,2} \cdot$ Ligong Wang ${ }^{1,2}$}

Published online: 3 October 2020

(c) Springer Japan KK, part of Springer Nature 2020

\section{Correction to: Graphs and Combinatorics https://doi.org/10.1007/s00373-020-02194-5}

In the original publication of the article, few errors have occurred. The corrections are given below:

1. Page 3, Theorem 2:

$$
g r_{k}\left(K_{3}: F_{9}\right)=g r_{k}\left(K_{3}: F_{10}\right)=\left\{\begin{array}{l}
8 \cdot 5^{(k-2) / 2}+1, \\
4 \cdot 5^{(k-1) / 2}+1
\end{array}\right.
$$

should be

$$
g r_{k}\left(K_{3}: F_{9}\right)=g r_{k}\left(K_{3}: F_{10}\right)= \begin{cases}8 \cdot 5^{(k-2) / 2}+1, & \text { if } k \text { is even, } \\ 4 \cdot 5^{(k-1) / 2}+1, & \text { if } k \text { is odd }\end{cases}
$$

2. Page 3, Theorem 4 (3):

$$
k(n-1)+2 \geq g r_{k}\left(K_{3}: F_{2, n}\right) \geq\left\{\begin{array}{l}
\frac{5 n}{2}+k-6, \\
\frac{5 n-1}{2}+k-4,
\end{array}\right.
$$

The original article can be found online at https://doi.org/10.1007/s00373-020-02194-5.

Ligong Wang

lgwangmath@163.com

Xihe Li

1xhdhr@163.com

1 School of Mathematics and Statistics, Northwestern Polytechnical University, Xi'an 710129, Shaanxi, People's Republic of China

2 Xi'an-Budapest Joint Research Center for Combinatorics, Northwestern Polytechnical University, Xi' an 710129, Shaanxi, People's Republic of China 
should be

$$
k(n-1)+2 \geq g r_{k}\left(K_{3}: F_{2, n}\right) \geq \begin{cases}\frac{5 n}{2}+k-6, & \text { if } n \text { is even, } \\ \frac{5 n-1}{2}+k-4, & \text { if } n \text { is odd. }\end{cases}
$$

3. Page 4, Theorem 5:

$$
g r_{k}\left(K_{3}: F_{12}\right)=g r_{k}\left(K_{3}: F_{13}\right)=\left\{\begin{array}{l}
9 \cdot 5^{(k-2) / 2}+1, \\
4 \cdot 5^{(k-1) / 2}+1,
\end{array}\right.
$$

should be

$$
g r_{k}\left(K_{3}: F_{12}\right)=g r_{k}\left(K_{3}: F_{13}\right)= \begin{cases}9 \cdot 5^{(k-2) / 2}+1, & \text { if } k \text { is even, } \\ 4 \cdot 5^{(k-1) / 2}+1, & \text { if } k \text { is odd. }\end{cases}
$$

4. Page 5, Lemma 2:

$$
g r_{k}\left(K_{3}: K_{3}\right)=\left\{\begin{array}{l}
5^{k / 2}+1, \\
2 \cdot 5^{(k-1) / 2}+1,
\end{array}\right.
$$

should be

$$
g r_{k}\left(K_{3}: K_{3}\right)= \begin{cases}5^{k / 2}+1, & \text { if } k \text { is even } \\ 2 \cdot 5^{(k-1) / 2}+1, & \text { if } k \text { is odd }\end{cases}
$$

5. Page 5, Lemma 3:

$$
g r_{k}\left(K_{3}: F_{9}\right)>\left\{\begin{array}{l}
8 \cdot 5^{(k-2) / 2}, \\
4 \cdot 5^{(k-1) / 2}
\end{array},\right.
$$

should be

$$
g r_{k}\left(K_{3}: F_{9}\right)> \begin{cases}8 \cdot 5^{(k-2) / 2}, & \text { if } k \text { is even, } \\ 4 \cdot 5^{(k-1) / 2}, & \text { if } k \text { is odd }\end{cases}
$$

6. Page 5, Lemma 4:

$$
g r_{k}\left(K_{3}: F_{10}\right) \leq\left\{\begin{array}{l}
8 \cdot 5^{(k-2) / 2}+1 \\
4 \cdot 5^{(k-1) / 2}+1
\end{array}\right.
$$


should be

$$
g r_{k}\left(K_{3}: F_{10}\right) \leq \begin{cases}8 \cdot 5^{(k-2) / 2}+1, & \text { if } k \text { is even } \\ 4 \cdot 5^{(k-1) / 2}+1, & \text { if } k \text { is odd }\end{cases}
$$

7. Page 5, Proof of lemma 4:

$$
n=\left\{\begin{array}{l}
8 \cdot 5^{(k-2) / 2}+1 \\
4 \cdot 5^{(k-1) / 2}+1
\end{array}\right.
$$

should be

$$
n= \begin{cases}8 \cdot 5^{(k-2) / 2}+1, & \text { if } k \text { is even, } \\ 4 \cdot 5^{(k-1) / 2}+1, & \text { if } k \text { is odd }\end{cases}
$$

8. Page 6:

$$
g r_{k-1}\left(K_{3}: K_{3}\right)=\left\{\begin{array}{l}
2 \cdot 5^{(k-2) / 2}+1, \\
5^{(k-1) / 2}+1
\end{array}\right.
$$

should be

$$
g r_{k-1}\left(K_{3}: K_{3}\right)= \begin{cases}2 \cdot 5^{(k-2) / 2}+1, & \text { if } k \text { is even } \\ 5^{(k-1) / 2}+1, & \text { if } k \text { is odd }\end{cases}
$$

9. Page 9:

$$
\left|V\left(G_{k}\right)\right|=\left\{\begin{array}{l}
\frac{5 n}{2}+k-7 \\
\frac{5 n-1}{2}+k-5
\end{array}\right.
$$

should be

$$
\left|V\left(G_{k}\right)\right|= \begin{cases}\frac{5 n}{2}+k-7, & \text { if } n \text { is even } \\ \frac{5 n-1}{2}+k-5, & \text { if } n \text { is odd. }\end{cases}
$$

10. Page 9:

$$
n_{k}=\left\{\begin{array}{l}
r_{2}\left(F_{2, n}\right)+k-2 \\
k+9 \\
k(n-1)+2
\end{array}\right.
$$


should be

$$
n_{k}= \begin{cases}r_{2}\left(F_{2, n}\right)+k-2, & \text { if } n \in\{3,4\} \text { and } k \geq 1, \\ k+9, & \text { if } n=5 \text { and } k \geq 2, \\ k(n-1)+2, & \text { if } n \geq 6 \text { and } k \geq 2\end{cases}
$$

11. Page 11, Lemma 6:

$$
g r_{k}\left(K_{3}: H\right)>\left\{\begin{array}{l}
9 \cdot 5^{(k-2) / 2}, \\
4 \cdot 5^{(k-1) / 2},
\end{array}\right.
$$

should be

$$
g r_{k}\left(K_{3}: H\right)> \begin{cases}9 \cdot 5^{(k-2) / 2}, & \text { if } k \text { is even, } \\ 4 \cdot 5^{(k-1) / 2}, & \text { if } k \text { is odd. }\end{cases}
$$

12. Page 11-12, Lemma 7:

$$
\operatorname{gr}_{k}\left(K_{3}: H\right) \leq\left\{\begin{array}{l}
9 \cdot 5^{(k-2) / 2}+1, \\
4 \cdot 5^{(k-1) / 2}+1
\end{array}\right.
$$

should be

$$
g r_{k}\left(K_{3}: H\right) \leq \begin{cases}9 \cdot 5^{(k-2) / 2}+1, & \text { if } k \text { is even, } \\ 4 \cdot 5^{(k-1) / 2}+1, & \text { if } k \text { is odd. }\end{cases}
$$

13. Page 12, Proof of Lemma 7:

$$
n=\left\{\begin{array}{l}
9 \cdot 5^{(k-2) / 2}+1 \\
4 \cdot 5^{(k-1) / 2}+1
\end{array}\right.
$$

should be

$$
n= \begin{cases}9 \cdot 5^{(k-2) / 2}+1, & \text { if } k \text { is even } \\ 4 \cdot 5^{(k-1) / 2}+1, & \text { if } k \text { is odd. }\end{cases}
$$

The original article has been corrected.

Publisher's Note Springer Nature remains neutral with regard to jurisdictional claims in published maps and institutional affiliations. 